\title{
Erosion of lithium coatings on TZM molybdenum and graphite during high-flux plasma bombardment
}

\author{
T. Abrams ${ }^{1,{ }^{*}}$, M.A. Jaworski ${ }^{1}$, R. Kaita ${ }^{1}$, D.P. Stotler ${ }^{1}$, G. De Temmerman ${ }^{2}$, T.W. Morgan ${ }^{2}$, \\ M.A. van den Berg ${ }^{2}$, H.J. van der Meiden ${ }^{2}$ \\ ${ }^{1}$ Princeton Plasma Physics Laboratory, Princeton, NJ, 08543, USA. \\ ${ }^{2}$ FOM Institute DIFFER-Dutch Institute For Fundamental Energy Research, Trilateral Euregio \\ Cluster, Associate EURATOM-FOM, BL-3430 BE Nieuwegein, The Netherlands.
}

\begin{abstract}
The rate at which Li films will erode under plasma bombardment in the NSTX-U divertor is currently unknown. It is important to characterize this erosion rate so that the coatings can be replenished before they are completely depleted. An empirical formula for the Li erosion rate as a function of deuterium ion flux, incident ion energy, and Li temperature was developed based on existing theoretical and experimental work. These predictions were tested on the Magnum-PSI linear plasma device capable of ion fluxes $>10^{24} \mathrm{~m}^{-2} \mathrm{~s}^{-1}$, ion energies of $20 \mathrm{eV}$ and $\mathrm{Li}$ temperatures $>800{ }^{\circ} \mathrm{C}$. Li-coated graphite and TZM molybdenum samples were exposed to a series of plasma pulses during which neutral Li radiation was measured with a fast camera. The total $\mathrm{Li}$ erosion rate was inferred from measurements of Li-I emission. The measured erosion rates are significantly lower than the predictions of the empirical formula. Strong evidence of fast Li diffusion into graphite substrates was also observed.
\end{abstract}

Keywords: lithium; lithium sputtering; lithium evaporation; lithium radiation; lithium plasma surface interaction.

\section{Introduction}

Many tokamak experiments have utilized thin-film lithium coatings in order to improve plasma performance, including NSTX [1], LTX [2], EAST [3], and FTU [4]. The application of thin-film boron coatings on carbon or molybdenum substrates is in use on Alcator C-Mod [5], DIII-D [6], ASDEX-U [7], and KSTAR [8], among others. Other thin films have been applied or proposed for use in tokamaks, including tin, Li-Sn eutectics, beryllium, and silicon. Coatings composed of the bulk wall material itself can also develop on the vessel walls over the course of the run campaign due to erosion and re-deposition from plasma bombardment $[9,10]$, a process known as material migration. Finally, low- $Z$ thin films have been proposed as a sacrificial layer [11] in order to protect the divertor from the heat fluxes $\geq 10 \mathrm{MW} / \mathrm{m}^{2}$ expected in power plant-scale fusion devices $[12,13]$. The crucial role that these coatings can play in both plasma performance and protection of the plasmafacing components (PFCs) motivates accurate characterization of their erosion rates so that these films can be replenished before they are completely depleted.

Thin Li and B coatings on graphite and TZM molybdenum substrates are planned as plasma-facing components in NSTX-U [14]. The NSTX-U divertor is expected to experience deuterium ion fluxes $\Gamma_{D+}>10^{23} \mathrm{~m}^{-2} \mathrm{~s}^{-1}$, D ion energies $E_{D+}<50 \mathrm{eV}$, and surface temperatures

\footnotetext{
* Corresponding author. Email: tabrams@pppl.gov. Phone: +1 (609) 243-2659.
} 
$T_{\text {surf }} \lesssim 800{ }^{\circ} \mathrm{C}[15,16]$. Previous experimental work $[17,18]$ has demonstrated that the gross Li erosion rate under D plasma bombardment is strongly dependent on $\Gamma_{D+}, E_{D+}$ and $T_{\text {surf }}$ due to evaporation and physical sputtering. These efforts, however, were limited to $T_{\text {surf }}<500{ }^{\circ} \mathrm{C}, E_{D+}<50 \mathrm{eV}$, and $\Gamma_{D+} \leq 10^{22} \mathrm{~m}^{-2} \mathrm{~s}^{-1}$ and thus are not directly applicable to NSTX-U. The goal of this paper is to predict the gross erosion rate of thin Li coatings in the NSTX-U divertor in order to predict how long these Li films will last. To this end, the specific NSTX-U divertor conditions listed above were closely replicated in the Magnum-PSI linear plasma device [19]. The resulting gross erosion rate of lithium-coated TZM molybdenum and graphite was then measured as a function of $\Gamma_{D+}$ and $T_{\text {surf }}$.

\section{Theory}

Consider a thin Li film with areal density $\rho$ under D plasma bombardment. The timedependence of $\rho$ is governed by the equation

$$
\frac{d \rho}{d t}=\Gamma_{\text {redep }}-\left(\Gamma_{\text {sputt }}+\Gamma_{\text {evap }}+\Gamma_{\text {diff }}\right)=(R-1)\left(Y \cdot \Gamma_{\mathrm{D}+}\right)
$$

where $\Gamma_{\text {redep }}, \Gamma_{\text {sputt }}, \Gamma_{\text {evap }}$, and $\Gamma_{\text {diff }}$ are the fluxes due to re-deposition, sputtering, evaporation, and diffusion, respectively. A schematic depiction of each of these processes is shown in Figure 1. The total "effective" erosion yield $Y$ (atoms/ion) is defined as $\left(\Gamma_{\text {sputt }}+\right.$ $\left.\Gamma_{\text {evap }}+\Gamma_{\text {diff }}\right) / \Gamma_{\mathrm{D}+}$ and the re-deposition fraction $R=\Gamma_{\text {redep }} /\left(\Gamma_{\text {sputt }}+\Gamma_{\text {evap }}+\Gamma_{\text {diff }}\right)$.

Physical Li sputtering yields at room temperature can be calculated with the TRIM Monte Carlo code [20], which predicts yields $Y_{\text {sputt }} \approx 0.003-0.03$ for $10-50 \mathrm{eV} \mathrm{D}$ ions at normal incidence [21]. It has been demonstrated experimentally [17] that approximately $2 / 3$ of the $\mathrm{Li}$ is sputtered as an ion. These ions are re-deposited within several Debye lengths of their original location because they cannot escape the sheath potential well. This process is known as prompt, local re-deposition [22]. This reduces the yield to only the atomic contribution $Y_{\text {sputt,atom }} \approx 0.001-0.01$. To good approximation sputtered atoms can be assumed to follow a $\cos \theta$ distribution, where $\theta$ is the angle from the surface normal [23]. Theory predicts that sputtered Li atoms follow a Thomson energy distribution [24] with a peak at half the surface binding energy $E_{B} / 2=0.84 \mathrm{eV}$ [21]. The measured average energy of $\mathrm{Li}$ atoms sputtered from solid coatings is approximately $1 \mathrm{eV}$ [18].

In order to model thermal dependence we decompose $Y_{\text {sputter }}$ into $Y_{\text {coll }}+Y_{\text {thermal }}$ where $Y_{\text {coll }}$ is the room-temperature component of the sputter yield modeled by binary collision approximation (BCA) codes like TRIM. $Y_{\text {thermal }}$ represents the sputter component from thermally-activated processes and is dependent on $T_{\text {surf }}$. Allain proposes the following empirical formula for thermal sputtering:

$$
Y_{\text {thermal }}\left(T_{\text {surf }}\right)=\frac{A}{\sqrt{k\left(T_{\text {surf }}+B\right)}} \exp \left[-\frac{U}{k\left(T_{\text {surf }}+B\right)}\right]
$$

where $U$ is the Li sublimation energy at melting point $(1.59 \mathrm{eV})$ and $k$ is Boltzmann's constant. $A$ and $B$ are empirical fitting parameters. 
The evaporative flux from the surface of liquid Li is commonly assumed to follow the Langmuir evaporation law [25]:

$$
\Gamma_{\text {evap }}\left(T_{\text {surf }}\right)=\frac{p_{L i}}{\sqrt{2 \pi m_{L i} k T_{\text {surf }}}}
$$

Here $m_{\mathrm{Li}}$ is the Li atomic mass (6.941 amu) and an empirical formula for the Li vapor pressure $p_{\mathrm{Li}}$ is given in [26]. Li atoms evaporate with a Maxwell-Boltzmann (thermal) energy distribution. As in the case of sputtering the angular profile is also well approximated by $\cos \theta$ [27]. The diffusive flux is given by Fick's law $\Gamma_{\text {diff }}=D(d n / d x)$, where $D$ is the diffusion coefficient and $n$ is the volumetric Li density (atoms $/ \mathrm{m}^{3}$ ). An empirical relation for the Li diffusion coefficient $D$ in graphite as a function of temperature is derived in [28]. Diffusion of lithium into refractory metals such as molybdenum is negligible relative to evaporation and sputtering.

Values for $Y_{\text {thermal }}$ are obtained by fitting the data in [18] to Equation 2, yielding fitting parameters $A \approx 0.016$ and $B \approx 350$. This results in the following expression for effective neutral atomic Li yield a Li-coated metal $(D=0)$ :

$$
Y=\frac{Y_{\text {coll }}}{3}+Y_{\text {thermal }}\left(T_{\text {surf }}\right)+\frac{\Gamma_{\text {evap }}\left(T_{\text {surf }}\right)}{\Gamma_{D+}}
$$

In Figure 2 we plot the total effective yield for a Li-coated metal vs. Li temperature at the plasma conditions of Doerner et al. as well as a higher-flux, lower-energy case $\left(\Gamma_{D+}=5^{*} 10^{23}\right.$ $\left.\mathrm{m}^{-2} \mathrm{~s}^{-1}, E_{D+}=20 \mathrm{eV}\right)$ more characteristic of Magnum-PSI. Yields in the high-flux case are systematically lower both due to the lower value of $Y_{\text {coll }}$ (resulting from lower $E_{D+}$ ) as well as the higher value of $\Gamma_{D+}$. This formula predicts that in the latter case Li evaporation will not be the dominant erosion mechanism for surface temperatures $<800{ }^{\circ} \mathrm{C}$.

\section{Experimental Measurements of Lithium Erosion 3.1 Experimental Apparatus and Procedure}

As discussed above the Magnum-PSI linear plasma device provides an ideal testing ground for measurements of Li erosion in the NSTX-U divertor regime. Details on the technical specifications of the device can be found in [19]. A cascaded-arc plasma source provides a method of exposing disc-shaped bare and Li-coated TZM molybdenum and ATJ graphite samples $2.5 \mathrm{~cm}$ in diameter to ion fluxes $\Gamma_{D+} \leq 10^{24} \mathrm{~m}^{-2} \mathrm{~s}^{-1}$ at electron densities $n_{e} \leq$ $8 \cdot 10^{20} \mathrm{~m}^{-3}$ and temperatures $T_{e} \leq 3 \mathrm{eV}$ in these discharges. Water-cooled copper coils provide an axial magnetic field $\leq 0.33 \mathrm{~T}$ normal to the sample surface for pulse durations $\leq 7$ s. NSTX typically operated with a toroidal magnetic field on-axis of $\sim 0.45 \mathrm{~T}$ and with NSTX-U this value will increase to $\sim 1.0 \mathrm{~T}$ [29]. A target holder capable of rotary motion provides the capability to independently expose up to five different samples without breaking vacuum, although only three were used in these experiments. All experiments described herein were conducted at a bias of $-20 \mathrm{~V}$ with respect to ground to provide incident ion energies of approximately $20 \mathrm{eV}$. A differential pumping system ensures that the neutral density near the target $n_{D} \leq 0.1 \cdot n_{e}$, which is consistent with simulations [30]. 
Thin coatings of $\mathrm{Li} \leq 1 \mu \mathrm{m}$ in thickness were applied in-situ using a prototype of the LITER evaporators [31] in use on NSTX. Coating thicknesses were derived by absolute calibration of the Li deposition rate recorded on a quartz crystal microbalance (QCM) as a function of the evaporator oven temperature. Typical deposition rates were in the range of $0.1-1 \mathrm{~nm} / \mathrm{s}$. The typical delay between the end of a Li evaporation cycle and the resumption of plasma operations was approximately 30 minutes, during which the Li coating was exposed to a residual background gas pressure of approximately $10^{-7}$ torr consisting primarily of water vapor as measured using a residual gas analyzer (RGA). While the results of [32] predict that the top several monolayers of the Li surface will be completely transform to lithium hydroxide $(\mathrm{LiOH})$ under these conditions, measurements from optical emission spectroscopy (OES) showed little to no evidence of O-I emission $(777 \mathrm{~nm})$ or $\mathrm{OH}$ emission (308-310 nm) in the plasma. This implies minimal hydroxide or atomic oxygen was present in the Li film.

The Magnum-PSI diagnostic suite was utilized to obtain detailed measurements of both the plasma and target surface. A depiction of the experiment and diagnostic setup is shown in Figure 3. Radial electron density and temperature profiles $n_{e}(r)$ and $T_{e}(r)$, where $r$ is the radial distance from the center of the plasma column, were obtained using a Thomson scattering (TS) system. Post-processed TS data for a typical Magnum-PSI pulse are shown in Figure 4. Spatially resolved neutral Li emission profiles were derived from a Phantom camera with a Li-I $(670.8 \mathrm{~nm})$ filter. A FLIR Systems infrared (IR) camera was calibrated against a spectrally resolved pyrometer to obtain emissivity-independent measurements of the target surface temperature, which ranged from room temperature up to approximately $800{ }^{\circ} \mathrm{C}$. Examples of fast camera and IR images are shown in Figure 5.

\subsection{Data Analysis}

The yield of neutral Li atoms $Y_{L i}$ was indirectly inferred from measurements of the line-integrated neutral lithium emission. The Li emission intensity (photons $/ \mathrm{m}^{3} \cdot \mathrm{s}$ ) is equal to $n_{\mathrm{Li}} n_{e} P_{\mathrm{Li}}$ where $P_{\mathrm{Li}}$ is the Li photon emissivity rate coefficient (PEC) obtained from the ADAS code package [33]. The neutral Li density $n_{\mathrm{Li}}$ in an inhomogeneous plasma can be calculated by solving the continuity equation in the near-surface plasma region:

$$
\frac{\partial n_{L i}}{d t}+\nabla \cdot\left(n_{L i} v_{L i}\right)=-n_{L i} n_{e} S+n_{L i+} n_{e} R+n_{L i+} n_{D} C
$$

where $S, R$, and $C$ represent the effective rate coefficients for electron impact ionization, recombination, and $\mathrm{D}-\mathrm{Li}^{1+}$ charge exchange $(\mathrm{CX})$, respectively. Recombination and ionization rate coefficients are obtained from ADAS and $\mathrm{D}-\mathrm{Li}^{1+}$ charge exchange rates are extrapolated from $[34,35]$. Plots of each rate coefficient versus $n_{e}$ and $T_{e}$ are shown in Figure 6. The relevant time and length scales for various plasma processes in Magnum-PSI are given in Table 1. It is evident that the ionization term dominates in this regime and the recombination and charge exchange terms can be neglected. In addition, the fast ionization of $\mathrm{Li}$ atoms indicates an equilibrium state $\partial n_{\mathrm{Li}} / \partial t \approx 0$ can be assumed. Finally, the high ratio $\lambda_{\text {coll }} / \lambda_{\text {ionize }} \geq 100$ implies that $\left|v_{\mathrm{Li}}\right|$ is essentially constant in time. As an approximation, monoenergetic Li ejection at $1 \mathrm{eV}$ (the average energy measured in [18]) is assumed. Results were highly insensitive to the value of $v_{\mathrm{Li}}$. 
Following the treatment of [36] the ion flux to the target is given by $\Gamma_{D+}=\frac{1}{2} n_{e} c_{S}$ where $c_{S}=\left(2 T_{e} / m_{D}\right)^{1 / 2}$ is the ion sound speed. The factor of 2 is present because the Magnum-PSI plasma is sufficiently collisional such that $T_{e}=T_{D+}$. Under these assumptions Equation 5 can be solved numerically for $n_{L i}$ given the boundary condition $\Gamma_{L i}=n_{L i} v_{L i}=$ $Y \Gamma_{D+}$ at the target. If all $\mathrm{Li}$ atoms were ejected perpendicular to the surface into a homogenous plasma, this equation could be solved analytically in 1-D for neutral Li density:

$$
n_{L i}(z)=\frac{Y \Gamma_{D+}}{v_{L i}} \exp \left(-\frac{Z}{\lambda_{\text {ionize }}}\right)
$$

where $z$ is the distance from the target surface. In practice, however, Equation 5 must be solved numerically in $2 \mathrm{D}$ for $n_{\mathrm{Li}}$ due to the cosine distribution of ejected $\mathrm{Li}$ atoms and inhomogeneous D plasma background. The procedure for this is as follows. A 2D grid is created with spacing $\Delta r=1 \mathrm{~mm}$ and $\Delta z=0.5 \mathrm{~mm}$ in the radial and axial directions respectively. Finer grid spacing in either $r$ or $z$ did not result in any appreciable differences in the solution. No $\phi$ dependence is present due to the azimuthal symmetry of the plasma column.

The coefficients $n_{L i}^{i i^{\prime} j}$, the contribution to the Li density at radial grid point $i$ and axial grid point $j$ due to Li erosion from the target at radial grid point $i^{\prime}$, are calculated by numerically solving Equation 5 over the entire $i, j$ grid with boundary condition $\Gamma_{L i}^{i^{\prime}}=Y^{i^{\prime}} \Gamma_{D+}^{i^{\prime}}$. A model for the line-integrated emission $I^{i i^{\prime}}$ from radial grid point $i$ due to Li erosion from radial grid point $i^{\prime}$ is then obtained by summing in the axial direction over all $j$ subscripts:

$$
I^{i i^{\prime}}=\sum_{j} n_{L i}^{i i^{\prime} j} n_{e}^{i j} P^{i j}
$$

where $n_{e}^{i j}$ and $P^{i j}$ are the electron density and PEC at each grid point. The number of axial grid points $N_{j}=100$ such that the near-surface cutoff distance $z_{0}=N_{j} \Delta z=5 \mathrm{~cm}$. In practice the coefficients $I^{i i^{\prime}}$ are not sensitive to $z_{0}$ so long as $z_{0} \gg \lambda_{\text {ionize }}$.

It is evident from Equations 6 and 7 that the quantities $I^{i i^{\prime}}$ are linearly dependent on $Y^{i^{\prime}}$. We will refer to this constant of proportionality as $\alpha$ such that $I^{i i^{\prime}}=\alpha^{i i^{\prime}} Y^{i^{\prime}}$. This separates $I_{i i^{\prime}}$ into known quantities $\alpha^{i i^{\prime}}$ and unknown quantities $Y^{i^{\prime}}$ that we wish to determine in experiments. The total line-integrated emission $I^{i}$ from each radial grid point $i$ is modelled by the following system of equations:

$$
\left[\begin{array}{c}
I^{1} \\
I^{2} \\
\vdots \\
I^{N_{i}}
\end{array}\right]=\left[\begin{array}{cccc}
\alpha^{11} & \alpha^{12} & \cdots & \alpha^{1 N_{i}} \\
\alpha^{21} & \alpha^{22} & & \\
\vdots & & \ddots & \vdots \\
\alpha^{N_{i} 1} & & \cdots & \alpha^{N_{i} N_{i}}
\end{array}\right]\left[\begin{array}{c}
Y^{1} \\
Y^{2} \\
\vdots \\
Y^{N_{i}}
\end{array}\right]
$$

where $N_{i}$ is the number of radial grid points.

A model for $I^{i}$ is useful because it can be compared with experimental observations of line-integrated Li-I emission. Measurements of Li-I photons $/ \mathrm{m}^{2} \mathrm{~s}$ recorded on the Phantom 
camera were radially averaged in $1 \mathrm{~mm}$ wide rings concentric with the center of the plasma column to create the $I^{i}$ inputs for Equation 8. In other words $I^{i}$ is the average line-integrated Li-I intensity in radial bin $i$, where $i-1 \mathrm{~mm}<r<i \mathrm{~mm}$. In practice the center of the column and target differ by up to $5 \mathrm{~mm}$, thus emission measurements for $r \geq 7.5 \mathrm{~mm}$ originate partially on the clamping ring rather than the sample itself. To mitigate this source of error measurements of $I$ outside of $r=7 \mathrm{~mm}$ are discarded so the number of radial grid points $N_{i}=7$. Equation 8 is then solved for $Y^{i}$ by inverting the $\alpha^{i i^{\prime}}$ matrix and multiplying it by the measured values of $I^{i}$. It should also be noted that the line of sight of the Phantom camera is tilted $\sim 45^{\circ}$ from the surface normal. However the ratio of the decay length of the Li-I radiation intensity to the plasma column radius $\lambda_{\text {ionize }} / r_{0} \leq 0.1$. This implies that Li radiation is strongly localized near the target and the tilted line of sight of the camera does not introduce significant uncertainty into the measurements.

Plasma exposures on Li-coated graphite targets were conducted at lower $\Gamma_{D+}$ in order to prevent $T_{\text {surf }}$ from getting overly high $\left(\Gamma_{D_{+}} \leq 10^{23} \mathrm{~m}^{-2} \mathrm{~s}^{-1}, T_{\text {surf }}<400{ }^{\circ} \mathrm{C}\right)$. As a result, however, $\lambda_{\text {ionize }} / r_{0}>1$ and the radiation profile is significantly altered by the tilted viewing angle of the Phantom camera. Thus measured values of $I^{i}$ as a function of radial bin $i$ cannot easily be deduced. As an alternative we sum the total Li-I emission $I$ from the target and replace $Y^{i^{\prime}}$ with some "average" Li yield $\langle Y\rangle$ that is independent of $r$ :

$$
I=\sum_{i} \sum_{i^{\prime}} I^{i i^{\prime}}=\langle Y\rangle \sum_{i} \sum_{i^{\prime}} \alpha^{i i^{\prime}}
$$

This is a single equation that is solved for $\langle Y\rangle$ rather than the system of equations in (8) that was solved for $Y^{i}$ for all $i$. This method allows one to calculate the accumulated Li fluence from the substrate as a function of time $\Phi_{L i}(t)=\int_{0}^{t}\left\langle Y\left(t^{\prime}\right)\right\rangle\left\langle\Gamma_{D+}\left(t^{\prime}\right)\right\rangle d t^{\prime}$ where here the quantities $\left\langle Y\left(t^{\prime}\right)\right\rangle$ and $\left\langle\Gamma_{D+}\left(t^{\prime}\right)\right\rangle$ are now averaged over the target surface.

\section{Results and Discussion}

A plot of the inferred neutral-atom Li yield $Y_{\mathrm{Li}}$ as a function of the Li temperature is shown in Figure 7. Li temperatures are obtained by averaging IR camera data in the same concentric ring geometry described above. The inferred Li yields are significantly lower than those predicted using Equation 4 and will be discussed below. Also shown are the inferred Li yields using the more straightforward ionizations/photon (SXB) technique that has been used in other studies [37]. SXB coefficients are also obtained from the ADAS database. The SXB technique makes the assumptions that atomic excitation during ionization is the only source of photon emission and the Li impurity species is completely ionized along the line of sight of the diagnostic. These approximations are strongly satisfied in this particular experiment, and thus agreement with the SXB method is not surprising. If these same Li-I emission measurements originated from a vapor-shielded plasma target $\left(n_{e}=10^{21} \mathrm{~m}^{-3}, T_{e}=0.5 \mathrm{eV}\right)$, the yields inferred by these methods would differ by roughly a factor of 2 .

Inferred Li yields on Li-coated TZM are far lower than extrapolations from the empirical formula shown in Figure 7, which proven a fairly reliable predictor of Li erosion on 
numerous experiments. Therefore other mechanisms that could lead to a strong reduction in Li-I emission were investigated. The ratio Li-I photon mean free path $\lambda_{\text {absorb }}$ remains larger than the Li density decay length $\lambda_{\text {ionize }}$, indicating that radiation trapping is not a significant effect in this experiment $[38,39]$. Li-I emission intensity is also strongest in the center of the target where the highest Li densities are present, further indicating that the Li vapor cloud is not optically thick. Resonances do exist in the $\mathrm{Li}_{-}-\mathrm{Li}^{+}$charge exchange cross sections, but only at higher Li velocities than present here [40]. If Li recombination or charge exchange were significant additional sources of neutral $\mathrm{Li}$ atoms, a given $\mathrm{Li}-\mathrm{I}$ emission rate would correspond an even lower Li yield. Thus neglecting the recombination and CX gives an upper bound on the Li erosion rate. Finally, the processes of dielectronic recombination and collision-induced quenching of Li excitation are both accounted for in the ADAS collisionalradiative model [33].

This suggests that a novel Li suppression effect may be present in this particular experiment. One hypothesis proposed here for the strong observed reduction in Li erosion is Li interactions with $\mathrm{D}$ atoms. Liquid lithium on a high-Z substrate will retain atomic deuterium in the form of lithium deuteride (LiD) until a 1:1 stoichiometric ratio is achieved [41] and D-saturated Li layers demonstrate systematically lower sputtering yields than pure Li [17]. At the incident flux $\Gamma_{\mathrm{D}+}=10^{24} \mathrm{~m}^{-2} \mathrm{~s}^{-1}$ typical of Magnum-PSI, a $100 \mathrm{~nm}$ Li coating is exposed to a $\mathrm{D}$ fluence equal to the coating areal density $\rho_{L i}$ in $<20 \mathrm{~ms}$, suggesting that the $\mathrm{Li}$ layer is entirely $\mathrm{D}$-saturated for essentially the entire duration of plasma bombardment. This is in contrast to previous studies $[17,18]$ with orders of magnitude lower D fluxes and thicker Li layers, leading to much longer time scales for any D saturation effect to occur.

Another mechanism that may lead to suppression of Li erosion is interaction between the Li and the underlying TZM substrate. An effect is herein hypothesized in which Li-Mo bonding leads to an "effective binding energy" on the Li surface that exceeds the surface binding energy of pure $\mathrm{Li}$. In addition to chemical cohesion, $\mathrm{Li}$ is also capable of penetration into molybdenum along grain boundaries [42] and formation of Li-Mo-O compounds [43]. Recent surface science laboratory experiments [44] have demonstrated that material mixing occurs between Li and TZM Mo at a depth exceeding 10 monolayers $(3 \mathrm{~nm})$ as inferred from thermal desorption spectroscopy (TDS) measurements. The nature of this material mixing is still poorly understood but it suggests that modifications to the Li binding energy may be occurring.

A plot of the time-dependent Li fluence $\Phi_{\mathrm{Li}}$ from Li-coated TZM and Li-coated graphite is shown in Figure 8 relative to the initial coating areal density $\rho_{\mathrm{Li}}$ as determined from QCM measurements. The condition $\Phi_{\mathrm{Li}} / \rho_{\mathrm{Li}}=1$ corresponds to a $\mathrm{Li}$ layer that has completely eroded into the plasma the "worst-case scenario" of zero Li re-deposition. Both Li-coated samples demonstrate a decrease in erosion yield after the first 5-10 seconds. Because ion flux and Li surface temperature are fairly constant throughout the majority of each plasma discharge, no decrease in erosion yield is expected as long a Li coating exists on the surface of the sample. Thus a decreasing erosion rate suggests that a pure Li coating no longer exists to be eroded into the plasma. This Li depletion was verified by post-mortem visual inspection for both samples.

It is evident that the Li coating disappears from a graphite substrate much faster than expected from the measured Li erosion rate into the plasma. This suggests that fast diffusion 
of Li into graphite occurred. Previous analysis of Li-coated graphite tiles from NSTX showed evidence of $\mathrm{Li}$ up to 15 microns into the substrate, but not deeper than this [45]. Solving the 1-D equation for Li diffusion perpendicular to the basal planes of graphite, it is predicted that a $200 \mathrm{~nm}$ Li layer at room temperature will completely diffuse into the graphite substrate in < $5 \mathrm{~s}$. Nearly 30 minutes elapsed between the conclusion of Li evaporation onto graphite and the resumption of plasma exposures. Thus it is likely that a Li-C mixed material was present in the graphite target substrate as opposed to a pure Li surface coating available for erosion into the plasma. In contrast, the Li fluence from the Li-coated TZM sample strongly exceeds unity, indicating that significant re-deposition of eroded Li atoms is occurring.

\section{Conclusions}

An empirical formula for temperature-dependent Li erosion in the presence of D plasma bombardment was developed based on theory [17] and the results of a previous study [18]. This formula was extrapolated to the plasma and surface conditions present on Magnum-PSI and tested against a series of experiments involving bare and Li-coated TZM Mo and graphite. The inferred yields on Li-coated TZM Mo are significantly lower than the formula predicts. A $200 \mathrm{~nm} \mathrm{Li} \mathrm{coating} \mathrm{in} \mathrm{Magnum-PSI} \mathrm{is} \mathrm{observed} \mathrm{to} \mathrm{persist} \mathrm{for} \mathrm{several}$ seconds even at $\mathrm{D}$ ion fluxes exceeding $10^{24} \mathrm{~m}^{-2} \mathrm{~s}^{-1}$ and temperatures approaching $800{ }^{\circ} \mathrm{C}$. A mechanism involving the suppression of Li erosion via interactions with $\mathrm{D}$ or Mo was herein hypothesized. This strong discrepancy implies that predicted temperature limits [46] for Licoated high-Z PFCs in a tokamak are pessimistic.

In contrast, disappearance of the $\mathrm{Li}$ coating from the surface of $\mathrm{Li}$-coated graphite was observed after 1-2 seconds even at D fluxes $\Gamma_{D+} \approx 10^{23} \mathrm{~m}^{-2} \mathrm{~s}^{-1}$ and Li temperatures not exceeding $400{ }^{\circ} \mathrm{C}$. This is a strong indicator that fast $\mathrm{Li}$ diffusion into the bulk graphite substrate occurred. These results are consistent with the emprirical formula for Li diffusion [28] as well as the results of experiments on Li-coated graphite from NSTX [45]. If these results are parametrized in terms of the gross Li fluence $\Phi_{L i}$ divided by the initial areal density $\rho_{L i}$, a Li coating on TZM Mo is over 200 times more persistent than it is on Li-coated graphite. This implies that pure Li coatings on high- $Z$ substrates will last significantly longer than Li on graphite in the NSTX-U divertor.

\section{Acknowledgements}

This work is supported by US DOE contracts DE-AC02-09CH11466 and DE-AC0500OR22725. FOM authors are supported by the Stichting voor Fundamenteel Onderzoek der Materie (FOM), which is financially supported by the Nederlandse Organisatie voor Wetenschappelijk Onderzoek (NWO). Special thanks are extended to B.E. Koel, D.N. Ruzic, J.P. Allain for invaluable discussions concerning the interpretation of experimental data. 


\section{References}

[1] H.W. Kugel et al., J. Nucl. Mater. 415 (2011) S400-S404.

[2] J.C. Schmitt et al., J. Nucl. Mater. 438 (2013) S1096-S1099.

[3] G.Z. Zuo et al., J. Nucl. Mater. 415 (2011) S1062-S1066.

[4] G. Mazzitelli et al., Fusion Eng. Des. 85 (2010) 896-901.

[5] B. Lipschultz et al., J. Nucl. Mater. 363-365 (2007) 1110-1118.

[6] G.L. Jackson et al., J. Nucl. Mater. 196-198 (1992) 236-240.

[7] R. Neu et al., Fusion Eng. Des. 65 (2003) 367-374.

[8] S-H Hong et al., J. Nucl. Mater. 415 (2011) S1050-S1053.

[9] M. Rubel et al., J. Nucl. Mater. 313-316 (2003) 321-326.

[10] G.M. McCracken et al., J. Nucl. Mater. 145-147 (1987) 621-625.

[11] P.C. Stangeby and J. Davis, "Research required to develop the option of using carbon PFCs for application to high duty cycle tokamaks," University of Toronto, 2012.

[12] P. Norajitra et al., Fusion Eng. Des. 83 (2008) 893-902.

[13] A.S. Kukushkin et al., Nucl. Fusion 42 (2002) 187-191.

[14] R. Kaita et al., "NSTX-U Research Goals and Plans for Materials and Plasma Facing Components," APS-DPP Meeting, 2013.

[15] M.A. Jaworski et al., J. Nucl. Mater. 438 (2013) S384-S387.

[16] T.K. Gray et al., J. Nucl. Mater. 415 (2011) S360-S364.

[17] J.P. Allain et al., Phys. Rev. B 76, 205434 (2007).

[18] R.P. Doerner et al., J. Nucl. Mater. 290-293 (2001) 166-172.

[19] G. De Temmerman et al., Fus. Eng. Des. 88 6-8 (2013) 483-487.

[20] J.F. Ziegler, The Stopping Range of Ions in Matter, Vols. 2-6, Pergamon Press, 1977-1985.

[21] J. Laszlo and W. Eckstein, J. Nucl. Mater. 184 (1991) 22-29.

[22] P.C. Stangeby and A.W. Leonard, Nucl. Fusion 51 (2011) 1-16..

[23] M.D. Coventry, Ph.D. Thesis, "Temperature-enhanced low-energy ion sputtering of liquid tin: Measurements and modeling," University of Illinois Urbana-Champaign, 2007.

[24] M.W. Thompson, Philos. Mag. 18 (1968) 377-414..

[25] I. Langmuir, Mon. Weather Rev. 45 (1917) 452.

[26] APEX Interim Report, "Data Base for Liquid Breeders and Coolants," 1999.

[27] C.V. Deshpandey and R.F. Bunshah, Thin Film Processes, San Diego, 1991, pp. 79-132.

[28] N. Itou et al., J. Nucl. Mater. 290-293 (2001) 281-285.

[29] J.E. Menard et al., Nucl. Fusion 52 (2012) 083015.

[30] R.C. Wieggers et al., Contrib. Plasma Phys. 52 (2012) 440-444.

[31] H.W. Kugel et al., Phys. Plasmas 15 (2008) 056118.

[32] C.H. Skinner et al., J. Nucl. Mater. 438 (2013) S647-S650.

[33] H.P. Summers, "Atomic Data and Analysis Structure User Manual," 2004.

[34] R.K. Janev et al., J. Phys. B 29 (1996) 2497-2514.

[35] L.F. Errea et al., Phys. Rev A 77, 012706 (2008).

[36] P.C. Stangeby, The Plasma Boundary of Magnetic Fusion Devices, London: Taylor and Francis, 2000.

[37] J.P. Allain et al., Nucl. Fusion 44 (2004) 655-664.

[38] D.E. Post, J. Nucl. Mater. 220-222 (1995) 143-157.

[39] A. Kramida et al., "NIST Atomic Spectra Database," [Online]. Available: 
http://physics.nist.gov/asd. [Accessed 25 November 2013].

[40] J. Perel et al., Phys. Rev. Lett. 23 (1969) 677-680.

[41] M.J. Baldwin et al., Fusion Eng. Des. 61-62 (2002) 231-236.

[42] E.M. Lyutyi et al., Sov. Mater. Sci. 24 (1988) 357-361.

[43] W.H. McCarroll and M. Greenblatt, J. Solid State Chem. 54 (1984) 282-290.

[44] R. Sullengberger, M.S Thesis, "Uptake and Retention of Residual Vacuum Gases in Lithium and Lithium Films," Princeton University, 2012.

[45] W.R. Wampler et al., J. Nucl. Mater. 390-391 (2009) 1009-1012.

[46] T.D. Rognlien and M.E. Resnick, J. Nucl. Mater. 290-293 (2001) 312-316.

\section{Figure Captions}

1. Schematic depiction of the processes of sputtering, evaporation, re-deposition, and diffusion occurring during plasma bombardment of a thin film.

2. Plot of the evaporative and total atomic Li yields vs. Li temperature for the plasma conditions presented in [18] $\left(50 \mathrm{eV}, 8^{*} 10^{21} \mathrm{~m}^{-2} \mathrm{~s}^{-1}\right)$ as well as a high-flux, low-energy case $\left(20 \mathrm{eV}, 5^{*} 10^{23} \mathrm{~m}^{-2} \mathrm{~s}^{-1}\right)$ using Equation 4.

3. Depiction of the diagnostic setup in this experiment on Magnum-PSI.

4. Example electron density and temperature profiles in the Magnum-PSI plasma column, obtained from a single chord $2 \mathrm{~cm}$ from the target.

5. Example still camera images captured from the IR camera (top) and Phantom camera (bottom) at approximately a $45^{\circ}$ angle from the target surface.

6. Li ionization, recombination, and charge exchange rates vs. $n_{e}$ and $T_{e}$. Ionization and recombination rates are obtained from $\mathrm{ADAS}$ [33] and $\mathrm{D}-\mathrm{Li}^{1+} \mathrm{CX}$ rates are extrapolated from $[34,35]$. The range of electron temperatures covered in these experiments $\left(0.5 \mathrm{eV}<T_{e}<3 \mathrm{eV}\right)$ is shown with vertical lines.

7. Measured atomic Li yields vs. Li temperature. Predicted atomic yields using Equation 4 are also shown, as well as the TRIM yields for solid Li [21] and solid LiD sputtering, which have been divided by 3 to account for measurement of neutral atom sputtering only. Error bars indicate standard deviations.

8. Cumulative Li erosion fluence $\Phi_{\mathrm{Li}}$ relative to initial areal density $\rho_{\mathrm{Li}}$ for $\mathrm{Li}$-coated TZM and Li-coated graphite samples. The values in the graphite case have been magnified by a factor of 100 . The small discontinuities in both curves correspond to the ramp-up phase of the magnetic field at the start of each plasma exposure.

\section{Table Captions}

1. Typical values of various plasma parameters in Magnum-PSI during these experiments. 


\begin{tabular}{lll}
\hline Symbol & Quantity & Typical Value \\
\hline$S$ & Li ionization rate coefficient & $10^{-9}-10^{-7} \mathrm{~cm}^{-3} \mathrm{~s}^{-1}$ \\
$R$ & Li recombination rate coefficient & $10^{-13}-10^{-11} \mathrm{~cm}^{-3} \mathrm{~s}^{-1}$ \\
$C$ & $\mathrm{D}-\mathrm{Li}^{1+} \mathrm{CX}$ rate coefficient & $10^{-12}-10^{-11} \mathrm{~cm}^{-3} \mathrm{~s}^{-1}$ \\
$n_{L i}$ & Neutral Li density & $10^{12}-10^{13} \mathrm{~cm}^{-3}$ \\
$n_{L i+}$ & Li 1+ density & $10^{11}-10^{12} \mathrm{~cm}^{-3}$ \\
$n_{D}$ & Neutral deuterium density & $10^{13}-10^{14} \mathrm{~cm}^{-3}$ \\
$n_{e}$ & Electron density & $10^{14}-10^{15} \mathrm{~cm}^{-3}$ \\
$T_{e}$ & Electron temperature & $0.5-3 \mathrm{eV}$ \\
$v_{L i}$ & Ejected Li velocity & $52 \mathrm{~cm} / \mathrm{s}$ \\
$\lambda_{\text {coll }}$ & Li-D collisional mean free path & $10-300 \mathrm{~cm}$ \\
$\lambda_{\text {ionize }}$ & Li ionization mean free path & $0.03-0.1 \mathrm{~cm}$ \\
$\tau_{\text {ionize }}$ & Li ionization time & $1-5 \mu \mathrm{s}$ \\
$\lambda_{\text {absorb }}$ & Li-I photon mean free path & $0.1-1.0 \mathrm{~cm}$ \\
\hline
\end{tabular}

Table 1. Typical values of various plasma parameters in Magnum-PSI during these experiments. 


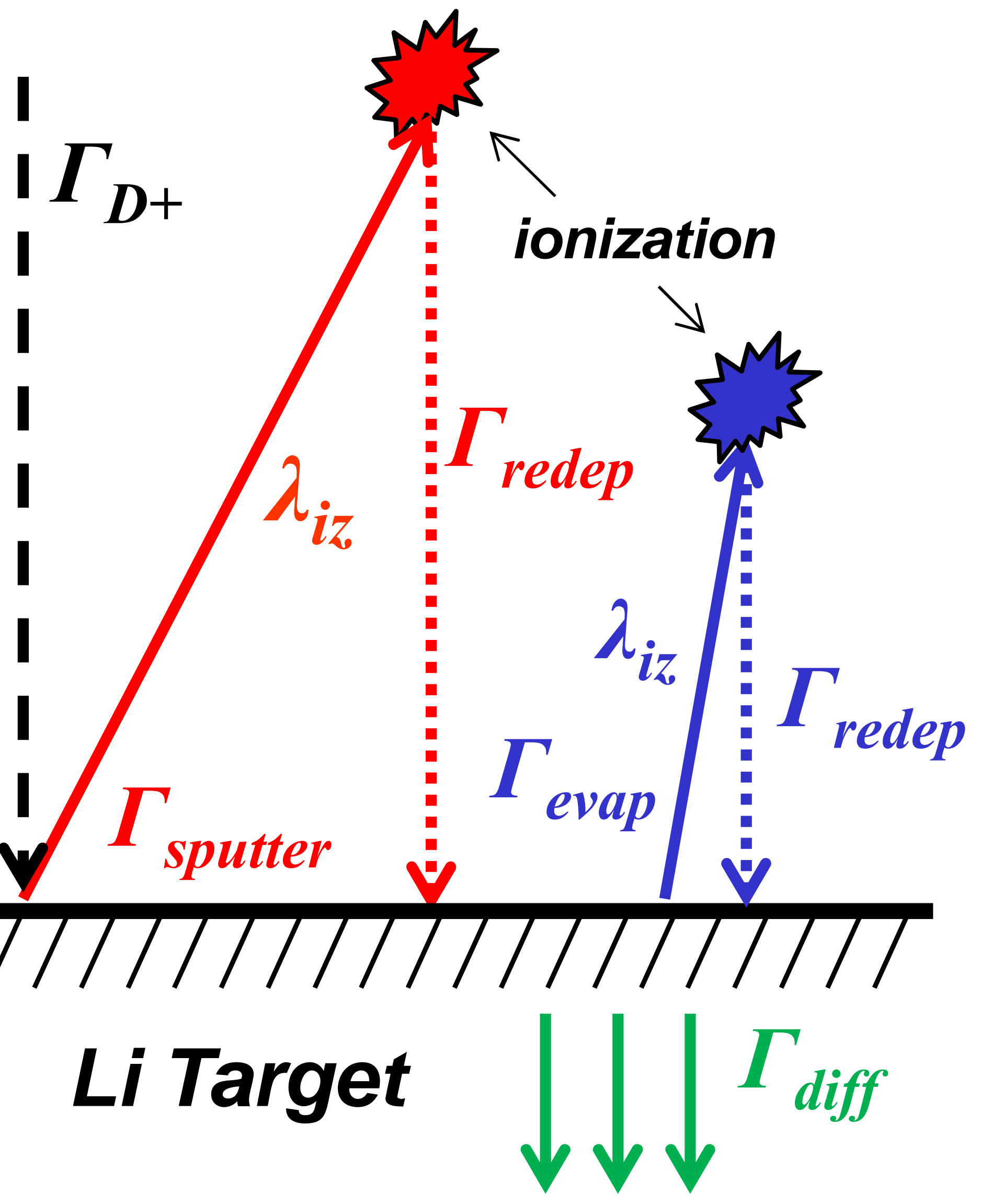




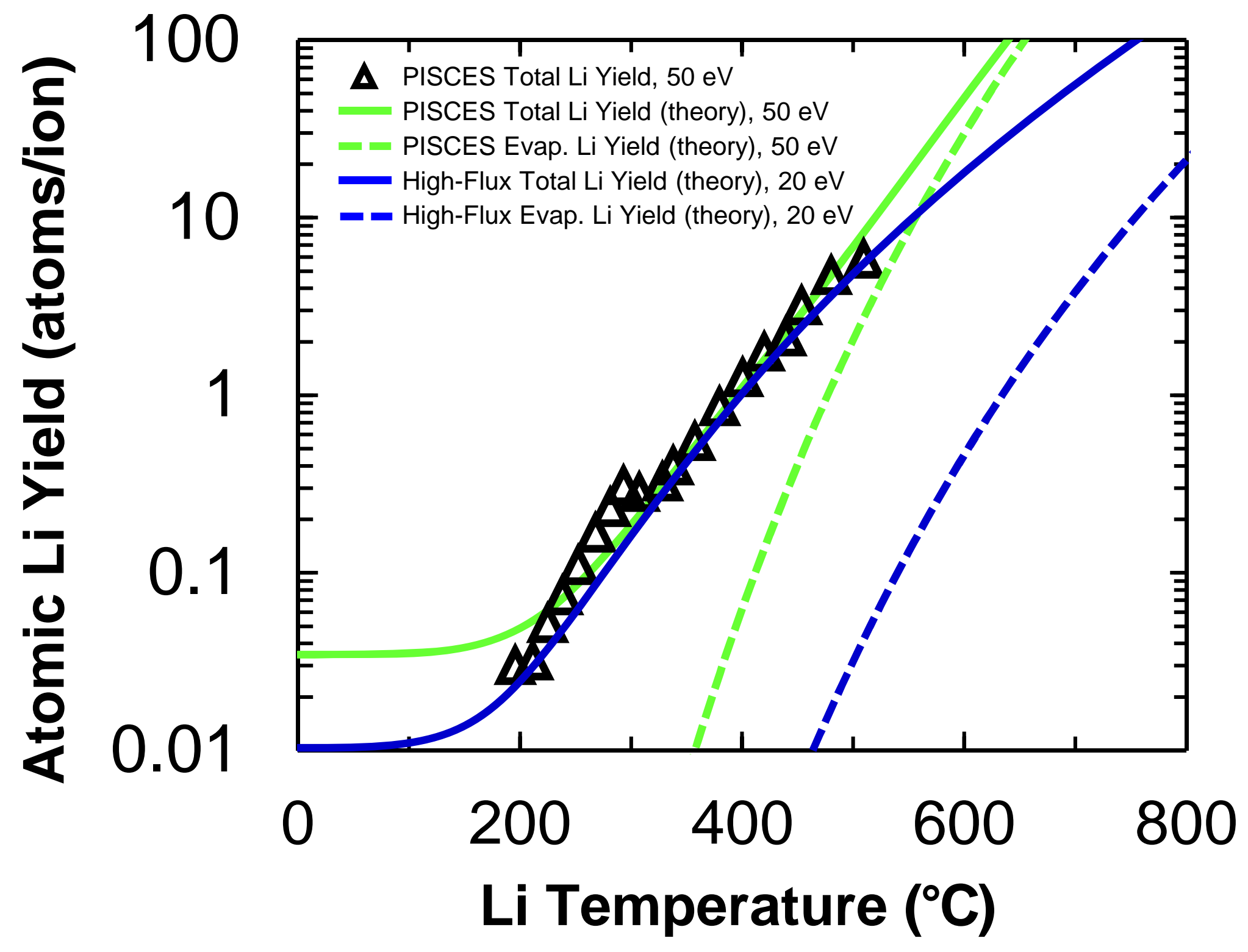




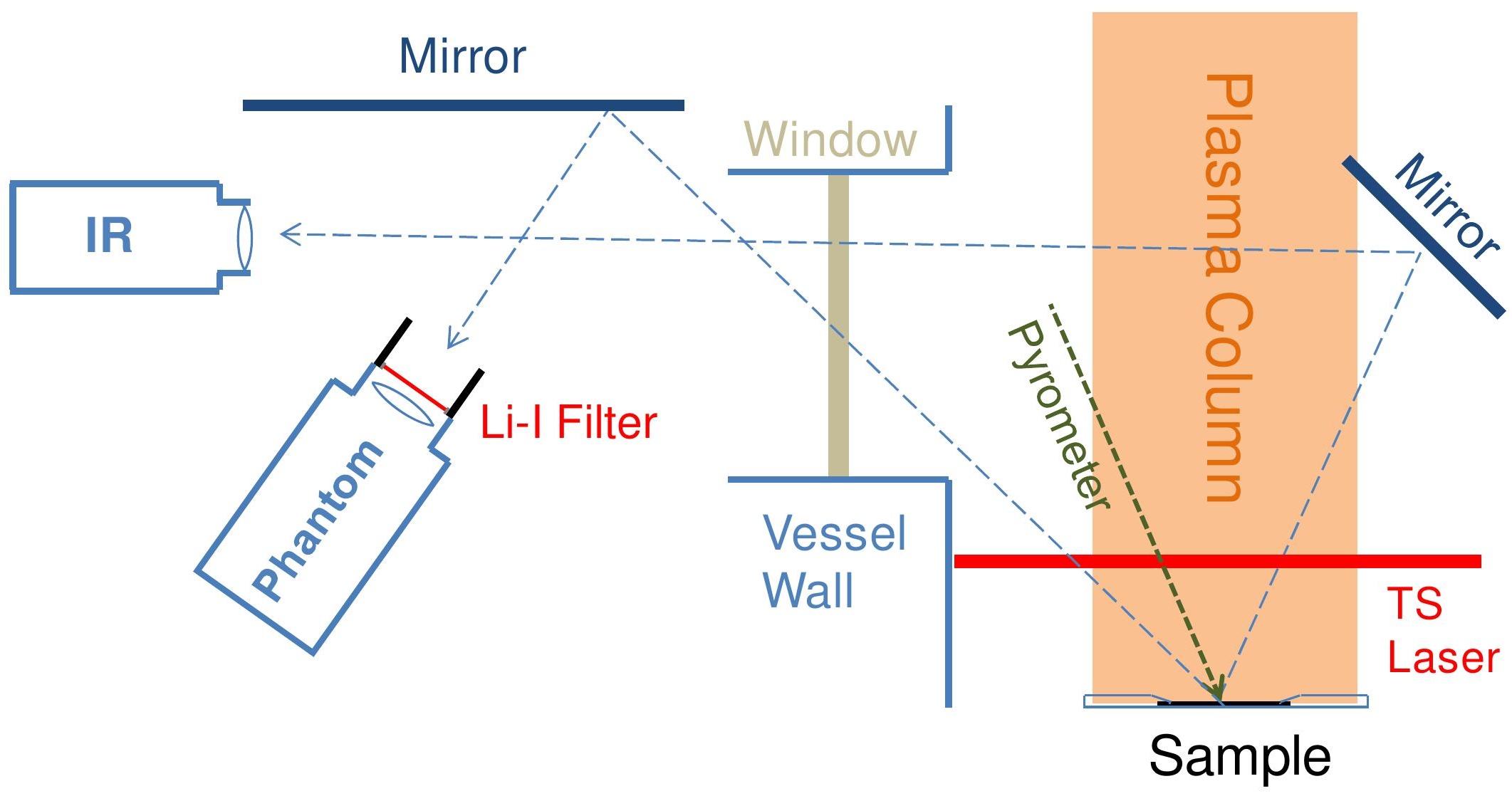




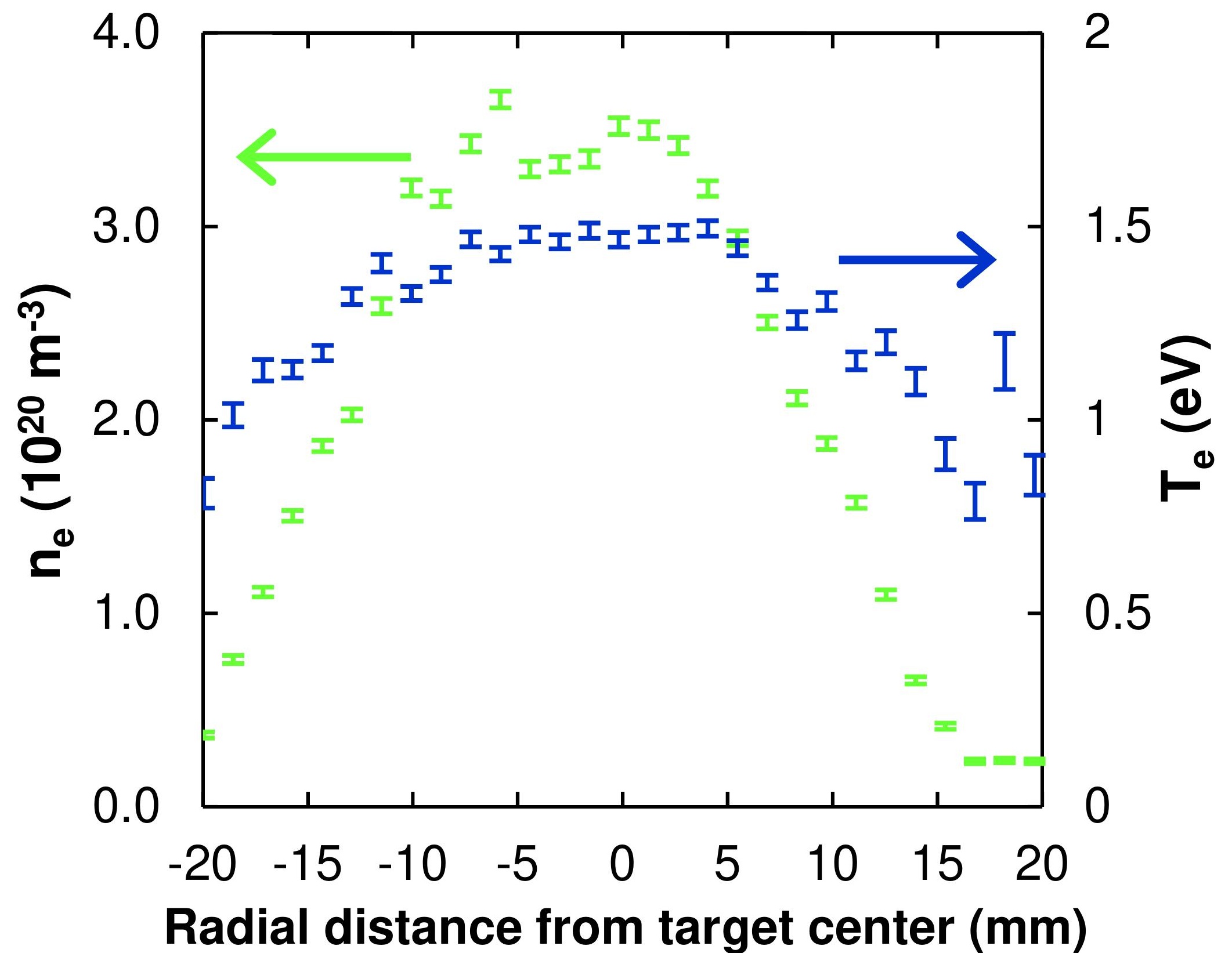




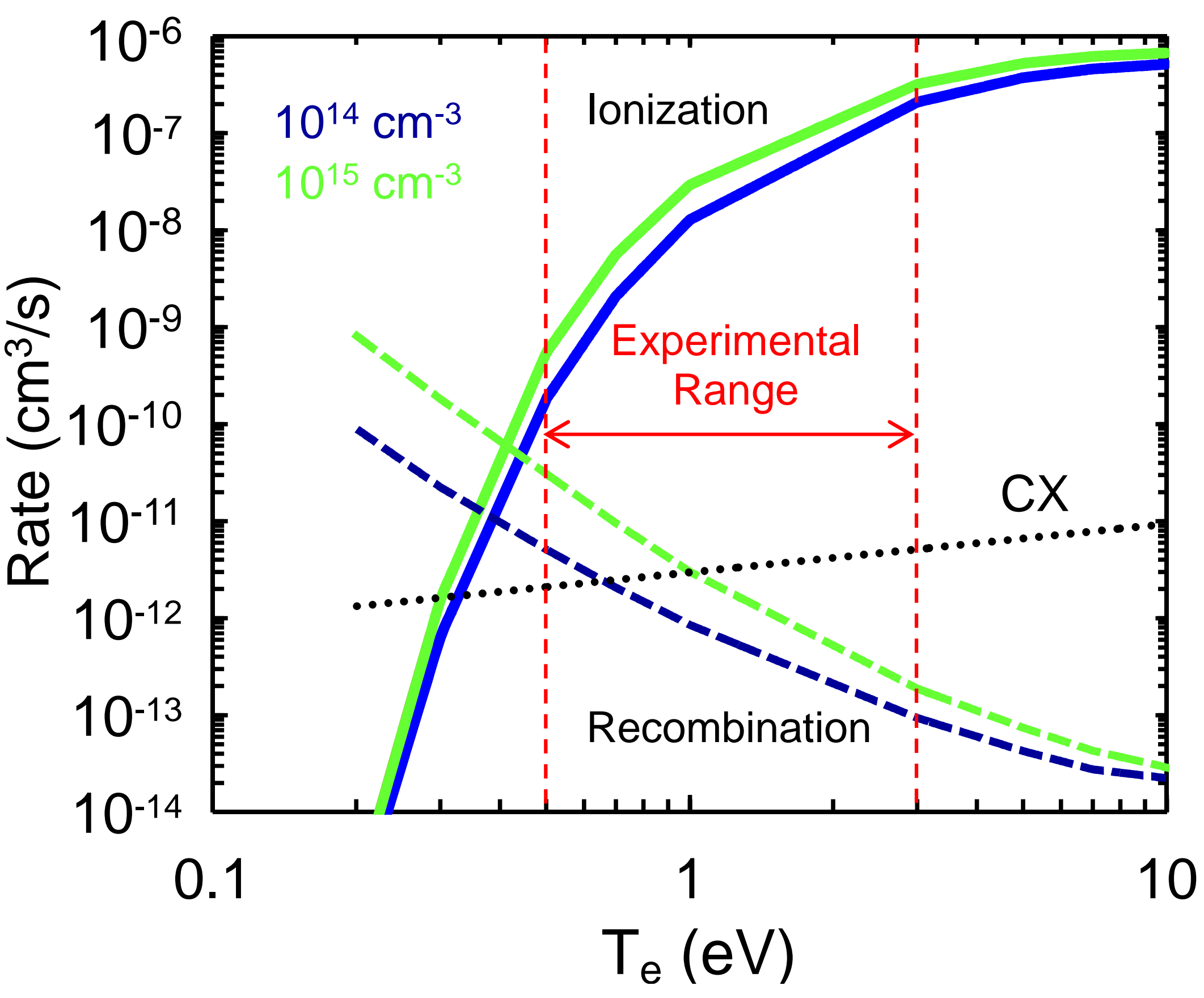




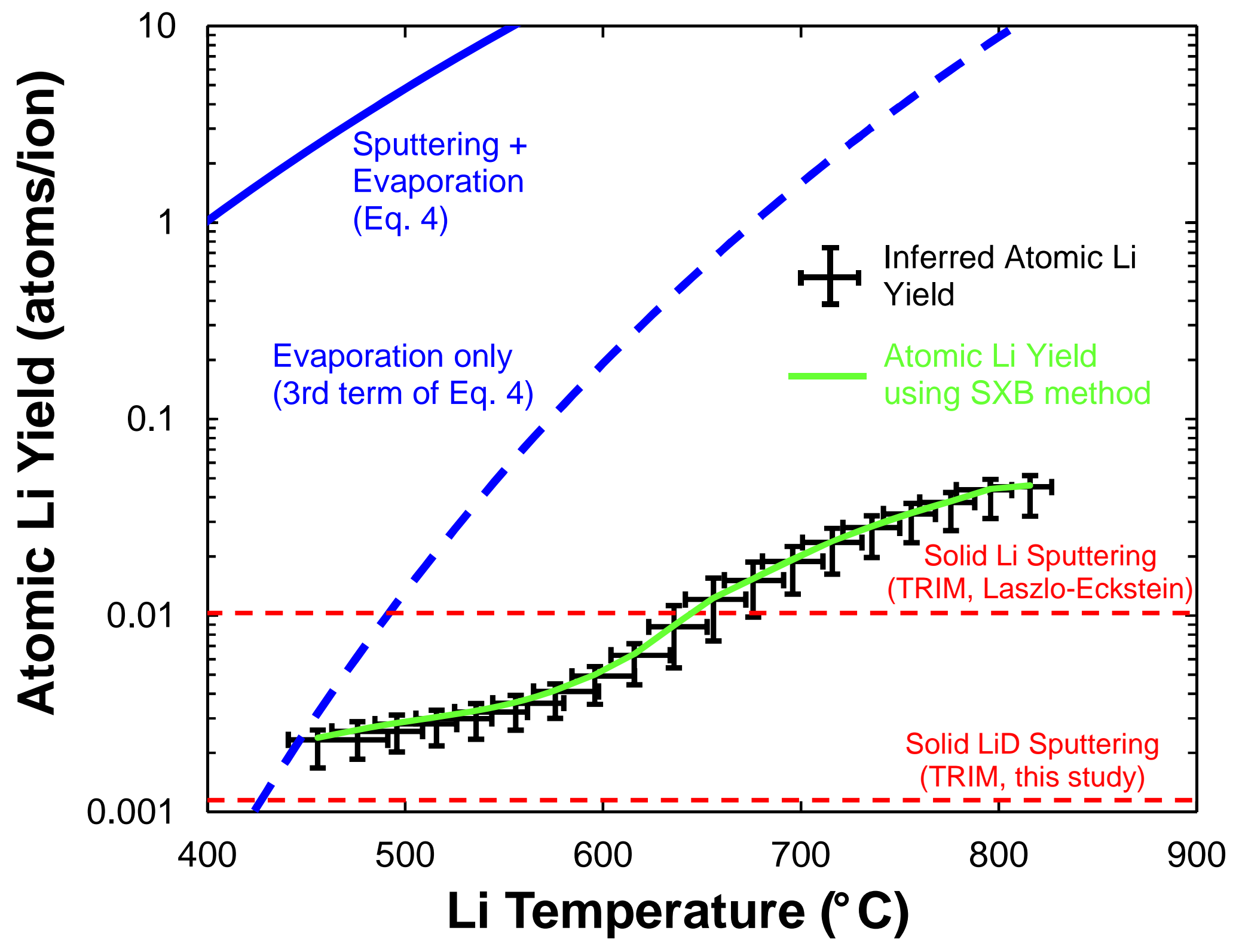




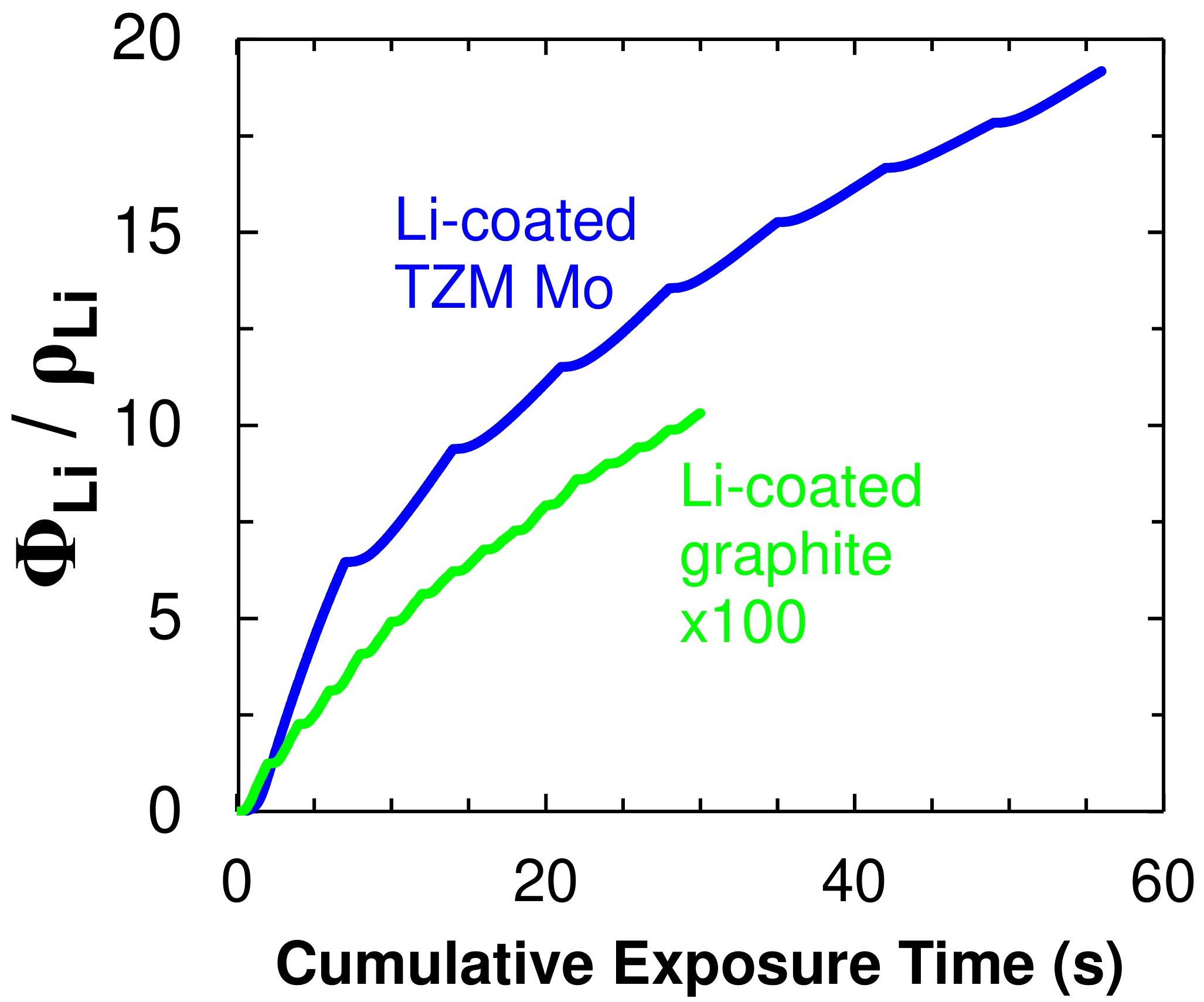

